\title{
The Influence of Ligand Side Chain on the Enantioselectivity of Lewis Acid Catalyzed Diels-Alder Reactions
}

\author{
Masashige Yamauchi, ${ }^{*}$ Kazuhiro ItaI, and Yuko Honda \\ Faculty of Pharmaceutical Sciences, Josai University; Keyakidai, Sakado, Saitama 350-0295, Japan. \\ Received May 7, 2002; accepted July 1, 2002
}

\begin{abstract}
The enantioselective Lewis acid-catalyzed Diels-Alder reaction of 3-(2-propenoyl)-1,3-oxazolidin-2-one 8 with cyclopentadiene was examined using a series of chiral mox ligands $2-6$, deferring in the side chain at 2-position of the chiral oxazoline and in the nature of the substituent at the chiral center (4-position) of the oxazoline ring, and a combination of $N$-[(1R)-2-chloro-1-phenylethyl]-2-[(4R)-4-phenyl-4,5-dihydrooxazol-2-yl]butyramide $2-\mathrm{MgI}_{2}-\mathrm{I}_{2}$ was the most efficient catalyst.
\end{abstract}

Key words enantioselective Diels-Alder reaction; Lewis acid; chiral mono(oxazoline) (mox); $N$-[(1R)-2-chloro-1-phenylethyl]2-[(4R)-4-phenyl-4,5-dihydrooxazol-2-yl]butyramide; 3-(2-propenoyl)-1,3-oxazolidin-2-one

The design and synthesis of novel chiral catalysts for asymmetric reactions continues to be an important and active area of research. ${ }^{1,2)} C_{2}$-symmetric bis(oxazolines) (boxes) have proven to be excellent ligands in wide range of asymmetric carbon-carbon bond formation reactions ${ }^{3}$ including Diels-Alder reaction. ${ }^{4-9)}$ Recently, a non- $C_{2}$-symmetric box bearing a meso backbone ${ }^{10)}$ and a non- $C_{2}$-symmetric mono(oxazoline) (mox) such as pyridyloxazoline ${ }^{11)}$ or pyosphinoaryloxazoline ${ }^{12-15)}$ have also been shown to be effective ligands in the enantioselective catalytic Diels-Alder reaction. In previous paper we have reported ${ }^{16,17)}$ that the magnesiumcatalyzed Diels-Alder reaction of ethyl 2-benzoylacrylate and 1-phenyl-2-methylenebutane-1,3-dione gave the adduct enantioselectively with $N$-[(1R)-2-chloro-1-phenylethyl]-2ethyl-2-[(4R)-4-phenyl-4,5-dihydrooxazol-2-yl]butyramide 2, a non- $C_{2}$-symmetric mox, as a chiral ligand. We have also proposed a plausible transition state like $\mathbf{1}$ in which hydrogen bonding between the chloride and the amide hydrogen of $\mathbf{2}$ plays an important role (Fig. 1). In order to test the influence of the side chain at 2-position of chiral oxazolines, we examined the Lewis acid-catalyzed Diels-Alder reactions of well-used 3-(2-propenoyl)-1,3-oxazolidin-2-one $\mathbf{8}^{18-22)}$ as a dienophile with cyclopentadiene using moxes $\mathbf{2}-\mathbf{6}$ as chiral ligands.

Moxes 3-6 were synthesized in almost the same manner as $2 .{ }^{17)}$ The Lewis acid-chiral ligand complex was prepared by refluxing a mixture of Lewis acid, chiral ligand and $\mathrm{I}_{2}$ at a ratio shown in Table 1 in $\mathrm{CH}_{3} \mathrm{CN}$. The solvent was removed and the resulting complex was dissolved in $\mathrm{CH}_{2} \mathrm{Cl}_{2}$ and cooled at temperature shown in Table 1. To this solution dienophile 8 in $\mathrm{CH}_{2} \mathrm{Cl}_{2}$ was added and stirred for $30 \mathrm{~min}$, then cyclopentadiene in $\mathrm{CH}_{2} \mathrm{Cl}_{2}$ was added slowly for a period of $3 \mathrm{~h}$. The reaction mixture was stirred for a period of time shown in Table 1. Usual work up gave the adduct. The endo/exo ratios were determined by ${ }^{1} \mathrm{H}-\mathrm{NMR}$ spectroscopy and the enantioselectivities were determined by analytical HPLC. The absolute configuration of the major endo-adduct in each reaction was determined by the comparison of retention time on HPLC and specific rotation with that reported for authentic sample. ${ }^{23)}$

Table 1 shows the results of the reaction using $\mathrm{MgI}_{2}(0.1$ eq) as Lewis acid. The enantiomeric excesses are dependent on the reaction temperature when the reactions were carried out with the complex prepared from $2: \mathrm{MgI}_{2}: \mathrm{I}_{2}(1: 1: 1)$ (entries 1,2$)$. On the contrary, no significant temperature dependencies were found in the reaction with the complex prepared from $2: \mathrm{MgI}_{2}: \mathrm{I}_{2}(2: 1: 2)$ (entries 3-5). At the same reaction temperature the enantiomeric excesses increased more than $20 \%$ by changing the complex composition $\left(2: \mathrm{MgI}_{2}: \mathrm{I}_{2}\right)$ from $(1: 1: 1)$ to $(2: 1: 2)$ (entries 1,3$)$. The high asymmetric induction in the reaction can be rationalized by assuming that the reaction proceeds via the intermediacy of a tetrahedral complex $\mathbf{1 0}$ shown in Fig. 2, which is similar to that of the reaction of $\mathbf{8}$ with $\mathrm{MgI}_{2}-\mathbf{7 a}$ proposed by Corey. ${ }^{5)}$ As reported in the previous paper it is considerably difficult to achieve chelation between magnesium and $\mathbf{2}$, two equivalents of chiral ligand and iodine to Lewis acid would facilitate to form the complex. When oxazoline nitrogen and amide carbonyl oxygen coordinate to magnesium and hydrogen bonding between the chloride and the amide hydrogen, the phenyl group of amide side-chain situate at nearly opposite side to the phenyl group at 4-position of oxazoline ring across magnesium. Thus cyclopentadiene should approaches from re face to the complex 10. Next, we examined the contribution of the side chain at 2 position of the oxazoline to the reaction. The remarkable depletion of ee was observed converting $\mathrm{Cl}$ to $\mathrm{H}$ (compare entries 3 and 6). This means that a group having hydrogen-bonding ability is necessary for high enantioselectivity. Furthermore the reaction did not proceed with 4 having non-chiral long chain substituent at 2 position under the same reaction condition (entry 7). This might be due to inhibition of flexible long chain for attack of cyclopentadiene to $\mathrm{Mg}-\mathbf{4}-\mathbf{8}$ complex. Finally, the reactions with mox having different substituents such as isopropyl and tert-butyl group at the chiral center of the oxazoline ring and the same side chain at 2-position as $\mathbf{2}$ were examined. As can be seen from entries 9 and 10 the enantioselectivities were very poor, which might be attributed to the steric hindrance of bulky group adjacent the amide group resulting in prevention of hydrogen bond formation in the reactive species.

As Evans reported the very high enantioselectivity in the reaction of $\mathbf{8}$ with $\mathbf{7 c - C u ( O T f})_{2}$ complex, ${ }^{6}$ we next examined the reaction with $\mathrm{Cu}(\mathrm{OTf})_{2}(0.1 \mathrm{eq})$ as a Lewis acid (Table 2). However both endo- and enantio-selectivities were lower than those with $\mathrm{MgI}_{2}$ and the opposite enantiomer was formed with $\mathbf{6 b}$ (entry 3 ). The opposite enantiomer should be formed 
Table 1. Enantioselective Diels-Alder Reaction of 8 with Cyclopentadiene Using $\mathrm{MgI}_{2}$ in $\mathrm{CH}_{2} \mathrm{Cl}_{2}$

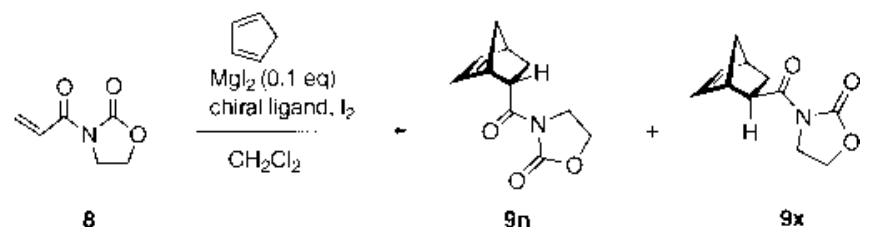

\begin{tabular}{|c|c|c|c|c|c|c|c|c|}
\hline \multirow{2}{*}{ Entry } & \multirow{2}{*}{$\begin{array}{l}\text { Ligand } \\
\text { (eq) }\end{array}$} & \multirow{2}{*}{$\begin{array}{c}\mathrm{I}_{2} \\
\text { (eq) }\end{array}$} & \multirow{2}{*}{$\begin{array}{l}\text { Temp. } \\
\left({ }^{\circ} \mathrm{C}\right)\end{array}$} & \multirow{2}{*}{$\begin{array}{l}\text { Time } \\
\text { (h) }\end{array}$} & \multirow{2}{*}{$\begin{array}{l}\text { Yield } \\
(\%)\end{array}$} & \multirow{2}{*}{$\begin{array}{c}\text { Ratio } \\
9 \mathbf{n}: \mathbf{9 x}\end{array}$} & \multicolumn{2}{|c|}{ endo-Isomer (9n) } \\
\hline & & & & & & & $\%$ ee & Config. \\
\hline 1 & $2(0.1)$ & 0.1 & -50 & 23 & 83 & $95: 5$ & 61.4 & $S$ \\
\hline 2 & $2(0.1)$ & 0.1 & -70 & 24 & 82 & $95: 5$ & 77.5 & $S$ \\
\hline 3 & $2(0.2)$ & 0.2 & -50 & 6 & 84 & $94: 6$ & 85.5 & $S$ \\
\hline 4 & $2(0.2)$ & 0.2 & -70 & 29 & 71 & $94: 6$ & 90.7 & $S$ \\
\hline 5 & $2(0.2)$ & 0.2 & -80 & 38 & 88 & $96: 4$ & 88.4 & $S$ \\
\hline 6 & $3(0.2)$ & 0.2 & -50 & 94 & 73 & $88: 12$ & 36.9 & $S$ \\
\hline 7 & $4 \quad(0.2)$ & 0.2 & -50 & 48 & - & - & - & - \\
\hline 8 & $5 \quad(0.2)$ & 0.2 & -70 & 17 & 80 & $95: 5$ & 3.2 & $R$ \\
\hline 9 & $\mathbf{6 a}(0.2)$ & 0.2 & -70 & 23 & 76 & $88: 12$ & 29.6 & $R$ \\
\hline 10 & $\mathbf{6 b}(0.2)$ & 0.2 & -70 & 24 & 78 & $93: 7$ & 18.5 & $R$ \\
\hline
\end{tabular}

Table 2. Enantioselective Diels-Alder Reaction of 8 with Cyclopentadiene Using $\mathrm{Cu}(\mathrm{OTf})_{2}$ in $\mathrm{CH}_{2} \mathrm{Cl}_{2}$

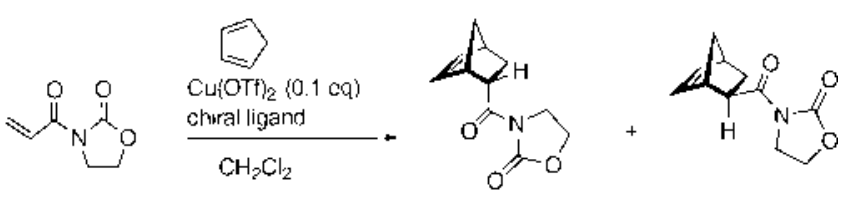

g 9n $9 \mathrm{x}$

\begin{tabular}{|c|c|c|c|c|c|c|c|}
\hline \multirow{2}{*}{ Entry } & \multirow{2}{*}{$\begin{array}{l}\text { Ligand } \\
\text { (eq) }\end{array}$} & \multirow{2}{*}{$\begin{array}{l}\text { Temp. } \\
\left({ }^{\circ} \mathrm{C}\right)\end{array}$} & \multirow{2}{*}{$\begin{array}{l}\text { Time } \\
\text { (h) }\end{array}$} & \multirow{2}{*}{$\begin{array}{l}\text { Yield } \\
(\%)\end{array}$} & \multirow{2}{*}{$\begin{array}{c}\text { Ratio } \\
\mathbf{9 n}: \mathbf{9 x}\end{array}$} & \multicolumn{2}{|c|}{ endo-Isomer (9n) } \\
\hline & & & & & & $\%$ ee & Config. \\
\hline 1 & $2(0.1)$ & -80 & 20 & 79 & $88: 12$ & 64.6 & $S$ \\
\hline 2 & $\mathbf{6 a}(0.1)$ & -80 & 46 & 65 & $81: 19$ & 6.2 & $R$ \\
\hline 3 & $\mathbf{6 b}(0.1)$ & -80 & 24 & 58 & $72: 28$ & 18.3 & $S$ \\
\hline
\end{tabular}

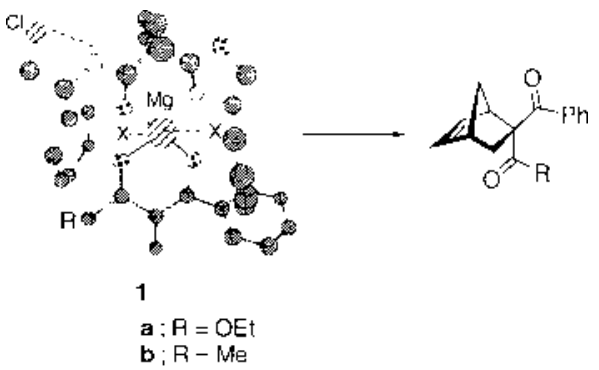

Fig. 1

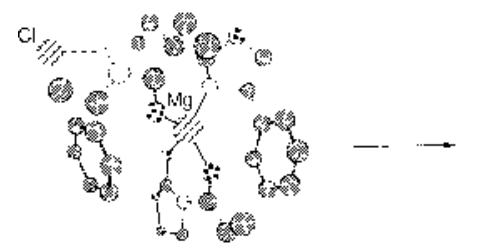

10

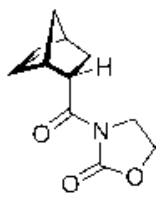

9n

Fig. 2

via a square-planar complex analogous to box $\mathbf{6 c - c o p p e r - 7}$ complex proposed by Evans. ${ }^{6,24)}$ or an octahedral complex ${ }^{22)}$ like 1.
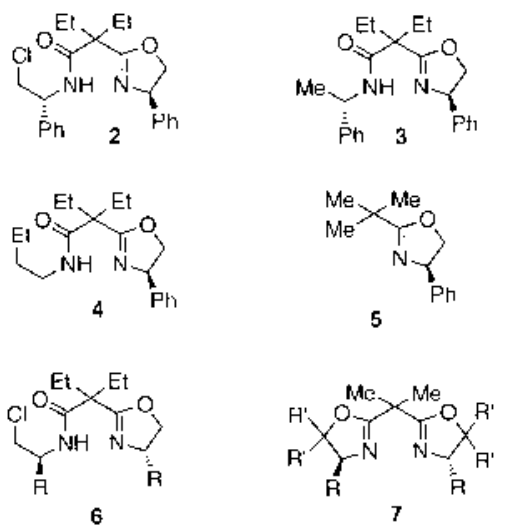

$$
a ; A=P r
$$$$
\boldsymbol{\theta} ; \mathrm{F}=\mathrm{Bu} !
$$

a, $\mathrm{A}=\mathrm{P}^{\prime}$. $\mathrm{A}^{\prime}=\mathbf{H}$

b: $R-P h, F^{\prime}=|M|$

c; $R=E \Delta^{\prime}, H^{\prime}=H$

Chart 1

In conclusion $N$-[(1R)-2-chloro-1-phenylethyl]-2-ethyl-2[(4R)-4-phenyl-4,5-dihydrooxazol-2-yl]butyramide 2 is also efficient ligand in the Diels-Alder reaction using 3-(2propenoyl)-1,3-oxazolidin-2-one 8 as a dienophile.

\section{Experimental}

Melting points are uncorrected. $\mathrm{MgSO}_{4}$ was used to dry organic layers 
after extraction. Column chromatography was performed with Silica Gel 60 (Spherical, Kanto Chemical Co.). HPLC was carried out with a Daicel Chiralcel OD column $(0.46 \times 25 \mathrm{~cm}$; eluate $0.1 \%$ propan- 2 -ol in hexane). NMR spectra were measured on a JEOL GX-270 spectrometer for samples in $\mathrm{CDCl}_{3}$ solution at $270 \mathrm{MHz}$ for ${ }^{1} \mathrm{H}$ and $67.89 \mathrm{MHz}$ for ${ }^{13} \mathrm{C}$, and chemical shifts are expressed in $\delta$-units using tetramethylsilane or chloroform as an internal standard. IR spectra were recorded on a JASXCO FT/IR-410 spectrometer. High-resolution mass spectra were obtained with a JEOL JMS-700 spectrometer.

Synthesis of Chiral Ligands. 2-Ethyl-2-[(4R)-4-phenyl-4,5-dihydrooxazol-2-yl]- $N$-[(1R)-1-phenylethyl]butyramide 3 To a solution of 2,2-diethyl- $N$-[(1R)-2-hydroxy-1-phenylethyl $]-N^{\prime}-[(1 R)$-1-phenylethyl]malonamide $(1.61 \mathrm{~g}, 4.2 \mathrm{mmol})$, and $\mathrm{Et}_{3} \mathrm{~N}(1.3 \mathrm{ml}, 9.24 \mathrm{mmol})$ in $\mathrm{CH}_{2} \mathrm{Cl}_{2}(50 \mathrm{ml})$ was added methanesulfonyl chloride $(0.36 \mathrm{ml}, 4.62 \mathrm{mmol})$ in an ice-bath. The reaction mixture was allowed to warm to room temperature and stirring was continued for $20 \mathrm{~min}$. The reaction mixture was then poured into saturated aqueous $\mathrm{NH}_{4} \mathrm{Cl}$ solution $(50 \mathrm{ml})$. The organic layer was separated and the aqueous layer was extracted with $\mathrm{CH}_{2} \mathrm{Cl}_{2}(20 \mathrm{ml} \times 2)$. The combined organic layers were washed with brine, dried and evaporated. The resulting crude mesylate was treated with $0.5 \mathrm{M} \mathrm{NaOH} / \mathrm{MeOH}-\mathrm{H}_{2} \mathrm{O}(1: 1)$ solution $(16 \mathrm{ml})$ at reflux for $1 \mathrm{~h}$. The reaction mixture was concentrated to half the original volume, and extracted with $\mathrm{CH}_{2} \mathrm{Cl}_{2}(15 \mathrm{ml})$. The organic layer was washed with brine $(10 \mathrm{ml})$, dried and evaporated. The resulting crystalline product was recrystallized from AcOEt-hexane to give $\mathbf{3}$ as colorless prisms; mp $106-108^{\circ} \mathrm{C}$; IR (KBr): 3210, $1660 \mathrm{~cm}^{-1}$; ${ }^{1} \mathrm{H}-\mathrm{NMR} \delta 0.72(3 \mathrm{H}, \mathrm{t}$, $J=7.3 \mathrm{~Hz}), 0.88(3 \mathrm{H}, \mathrm{t}, J=7.3 \mathrm{~Hz}), 1.47(3 \mathrm{H}, \mathrm{d}, J=7.0 \mathrm{~Hz}), 1.73-1.88(2 \mathrm{H}$, $\mathrm{m}), 2.03-2.18(2 \mathrm{H}, \mathrm{m}), 4.05(1 \mathrm{H}, \mathrm{t}, J=8.6 \mathrm{~Hz}), 4.66(1 \mathrm{H}, \mathrm{dd}, J=10.0$, $8.6 \mathrm{~Hz}), 5.20(1 \mathrm{H}$, quintet, $J=7.0 \mathrm{~Hz}), 5.36(1 \mathrm{H}$, br dd, $J=10.0,8.8 \mathrm{~Hz})$, $7.19-7.38(10 \mathrm{H}, \mathrm{m}), 10.41(1 \mathrm{H}$, brd, $J=7.5 \mathrm{~Hz}) .[\alpha]_{\mathrm{D}}^{18}-8.8^{\circ}(c=1.02$, $\mathrm{CHCl}_{3}$ ). Anal. Calcd for $\mathrm{C}_{23} \mathrm{H}_{28} \mathrm{~N}_{2} \mathrm{O}_{2}: \mathrm{C}, 75.79 ; \mathrm{H}, 7.74 ; \mathrm{N}, 7.69$. Found: $\mathrm{C}$, 75.83; H, 7.73; N, 7.69.

$N$-Butyl-2-ethyl-2-[(4R)-4-phenyl-4,5-dihydrooxazol-2-yl]butyramide 4 Compound 4 was synthesized from $N$-butyl-2,2-diethyl- $N^{\prime}-[(1 R)-2$-hydroxy-1-phenylethyl]malonamide $(8.0 \mathrm{~g}, 24 \mathrm{mmol})$ in a similar way as $\mathbf{3}$. Column chromatography $\left(0.5 \%\right.$ acetone in $\left.\mathrm{CH}_{2} \mathrm{Cl}_{2}\right)$ of the crude product gave $4(5.0 \mathrm{~g}, 66 \%)$ as a colorless viscous oil. IR (neat): $3250,1665 \mathrm{~cm}^{-1}$; ${ }^{1} \mathrm{H}-\mathrm{NMR} \delta 0.82(3 \mathrm{H}, \mathrm{t}, J=7.5 \mathrm{~Hz}), 0.86(3 \mathrm{H}, \mathrm{t}, J=7.0 \mathrm{~Hz}), 0.88(3 \mathrm{H}, \mathrm{t}$, $J=7.0 \mathrm{~Hz}), 1.33-1.40(2 \mathrm{H}, \mathrm{m}), 1.44-1.55(2 \mathrm{H}, \mathrm{m}), 1.72-1.90(2 \mathrm{H}, \mathrm{m})$, $2.04-2.19(2 \mathrm{H}, \mathrm{m}), 3.20-3.35(2 \mathrm{H}, \mathrm{m}), 4.05(1 \mathrm{H}, \mathrm{t}, J=8.6 \mathrm{~Hz}), 4.63(1 \mathrm{H}$, dd, $J=10.3,8.4 \mathrm{~Hz}), 5.31(1 \mathrm{H}, \mathrm{dd}, J=9.9,8.8 \mathrm{~Hz}), 7.24-7.41(5 \mathrm{H}, \mathrm{m}), 9.76$ $(1 \mathrm{H}$, br s $) \cdot[\alpha]_{\mathrm{D}}^{20}+14.2^{\circ}\left(c=1.06, \mathrm{CHCl}_{3}\right)$. High resolution electrom impact (HR-EI)-MS $m / z$ : 316.2148 (Calcd for $\mathrm{C}_{19} \mathrm{H}_{28} \mathrm{~N}_{2} \mathrm{O}_{2}: 316.2151$ ).

(4R)-2-tert-Butyl-4-phenyl-4,5-dihydrooxazol 5 To a solution of $N$ [(1R)-2-hydroxy-1-phenylethyl]-2,2-diethylpropionamide $(1.01 \mathrm{~g}, 4.6 \mathrm{mmol})$, and $\mathrm{Et}_{3} \mathrm{~N}(1.4 \mathrm{ml}, 10.1 \mathrm{mmol})$ in $\mathrm{CH}_{2} \mathrm{Cl}_{2}(45 \mathrm{ml})$ was added methanesulfonyl chloride $(0.36 \mathrm{ml}, 4.62 \mathrm{mmol})$ in an ice-bath. The reaction mixture was allowed to warm to room temperature and stirring was continued for $30 \mathrm{~min}$. The reaction mixture was then poured into saturated aqueous $\mathrm{NH}_{4} \mathrm{Cl}$ solution $(50 \mathrm{ml})$. The organic layer was separated and the aqueous layer was extracted with $\mathrm{CH}_{2} \mathrm{Cl}_{2}(20 \mathrm{ml} \times 2)$. The combined organic layers were washed with brine, dried and evaporated. The resulting crude mesylate was treated with $0.5 \mathrm{M} \mathrm{NaOH} / \mathrm{MeOH}-\mathrm{H}_{2} \mathrm{O}(1: 1)$ solution $(18 \mathrm{ml})$ at reflux for $1.5 \mathrm{~h}$. The reaction mixture was concentrated to half the original volume, and extracted with $\mathrm{CH}_{2} \mathrm{Cl}_{2}(20 \mathrm{ml} \times 3)$. The organic layer was washed with brine, dried and evaporated. The residue was subjected to column chromatography $\left(\mathrm{CHCl}_{3}\right)$ to give 5 as a colorless viscous oil. IR (neat): $1660 \mathrm{~cm}^{-1} ;{ }^{1} \mathrm{H}-\mathrm{NMR} \delta 1.31$ $(9 \mathrm{H}, \mathrm{s}), 4.05(1 \mathrm{H}, \mathrm{t}, J=8.0 \mathrm{~Hz}), 4.56(1 \mathrm{H}, \mathrm{dd}, J=10.5,8.3 \mathrm{~Hz}), 5.13(1 \mathrm{H}, \mathrm{dd}$, $J=10.5,7.8 \mathrm{~Hz}), 7.19-7.35(5 \mathrm{H}, \mathrm{m}) .[\alpha]_{\mathrm{D}}^{22}+87.9^{\circ}\left(c=4.12, \mathrm{CHCl}_{3}\right)$. HREI-MS $m / z$ : 203.1299 (Calcd for $\mathrm{C}_{13} \mathrm{H}_{27} \mathrm{NO}: 203.1310$ ).

$N$-[(1S)-1-Chloromethyl-2-methylpropyl]-2-ethyl-2-[(4S)-4-isopropyl4,5-dihydrooxazol-2-yl]butyramide 6 a To a solution of $N, N^{\prime}$-bis[(1S)-1chloromethyl-2-methylpropyl]-2,2-diethylmalonamide $(2.2 \mathrm{~g}, 6 \mathrm{mmol})$ in $\mathrm{MeOH}(130 \mathrm{ml})$ was added a solution of $\mathrm{NaOH}(240 \mathrm{mg}, 6 \mathrm{mmol})$ in $\mathrm{H}_{2} \mathrm{O}$ $(34 \mathrm{ml})$ at $60{ }^{\circ} \mathrm{C}$. The stirring was continued for $1 \mathrm{~h}$, and the reaction mixture was concentrated to $1 / 5$ the original volume. The organic layer was extracted with $\mathrm{CH}_{2} \mathrm{Cl}_{2}(50 \mathrm{ml} \times 3)$, dried and evaporated. The resulting residue was subjected to column chromatography $\left(0.5 \%\right.$ acetone in $\left.\mathrm{CH}_{2} \mathrm{Cl}_{2}\right)$ to give $\mathbf{6 a}$ $(1.2 \mathrm{~g}, 61 \%)$ as a colorless viscous oil. IR (neat): $3210,1670 \mathrm{~cm}^{-1} ;{ }^{1} \mathrm{H}-\mathrm{NMR}$ $\delta 0.76(3 \mathrm{H}, \mathrm{t}, J=7.3 \mathrm{~Hz}), 0.77(3 \mathrm{H}, \mathrm{t}, J=7.3 \mathrm{~Hz}), 0.95(3 \mathrm{H}, \mathrm{t}, J=7.0 \mathrm{~Hz})$, $0.98(3 \mathrm{H}, \mathrm{t}, J=7.0 \mathrm{~Hz}), 0.99(3 \mathrm{H}, \mathrm{t}, J=7.0 \mathrm{~Hz}), 1.00(3 \mathrm{H}, \mathrm{t}, J=7.0 \mathrm{~Hz})$, $1.69-1.80(3 \mathrm{H}, \mathrm{m}), 1.94-2.12(3 \mathrm{H}, \mathrm{m}), 3.59-3.73(2 \mathrm{H}, \mathrm{m}), 3.99-4.18$ $(3 \mathrm{H}, \mathrm{m}), 4.27(1 \mathrm{H}, \mathrm{dd}, J=8.8,7.3 \mathrm{~Hz}), 10.30(1 \mathrm{H}$, br d, $J=7.3 \mathrm{~Hz}) ;{ }^{13} \mathrm{C}-\mathrm{NMR}$ $\delta$ 9.7, 10.1, 18.2, 18.7, 18.9, 19.5, 28.4, 30.7, 31.6, 32.8, 45.9, 54.4, 55.5, 69.5, 72.2, 168.3, 171.5. $[\alpha]_{\mathrm{D}}^{24}-48.6^{\circ}\left(c=1.46, \mathrm{CHCl}_{3}\right)$. HR-EI-MS $m / z$ :
330.2066 (Calcd for $\mathrm{C}_{17} \mathrm{H}_{31} \mathrm{~N}_{2} \mathrm{O}_{2} \mathrm{Cl:}$ 330.2074).

2-[(4S)-4-tert-Butyl-4,5-dihydrooxazol-2-yl]- $N$-[(1S)-1-chloromethyl2,2-dimethylpropyl]-2-ethylbutyramide $6 \mathbf{b}$ The same treatment of $N, N^{\prime}$ bis[(1S)-1-chloromethyl-2,2-dimethylpropyl]-2,2-diethylmalonamide $(1.34 \mathrm{mg}, 3.4 \mathrm{mmol})$ gave crude product which was subjected to column chromatography $\left(0.5 \%\right.$ acetone in $\left.\mathrm{CH}_{2} \mathrm{Cl}_{2}\right)$ to give $\mathbf{6 b}(640 \mathrm{mg}, 46 \%)$ as a colorless viscous oil. IR (neat): $3210,1670 \mathrm{~cm}^{-1} ;{ }^{1} \mathrm{H}-\mathrm{NMR} \delta 0.79(3 \mathrm{H}, \mathrm{t}$, $J=7.1 \mathrm{~Hz}), 0.83(3 \mathrm{H}, \mathrm{t}, J=7.1 \mathrm{~Hz}), 0.93(9 \mathrm{H}, \mathrm{s}), 1.02(6 \mathrm{H}, \mathrm{s}), 1.67-1.81$ $(2 \mathrm{H}, \mathrm{m}), 1.97-2.17(2 \mathrm{H}, \mathrm{m}), 3.51(1 \mathrm{H}, \mathrm{dd}, J=11.4,8.0 \mathrm{~Hz}), 3.81(1 \mathrm{H}, \mathrm{dd}$, $J=11.4,3.3 \mathrm{~Hz}), 3.94-4.06(2 \mathrm{H}, \mathrm{m}), 4.13-4.25(2 \mathrm{H}, \mathrm{m}), 10.26(1 \mathrm{H}, \mathrm{brd}$, $J=8.5 \mathrm{~Hz}) ;{ }^{13} \mathrm{C}$-NMR $\delta 9.8,10.2,26.1,26.5,27.2,31.0,32.1,33.2,34.8$, $45.3,54.8,58.6,67.6,75.9,168.7,171.7 .[\alpha]_{\mathrm{D}}^{23}-7.7^{\circ}\left(c=1.30, \mathrm{CHCl}_{3}\right)$. HR-chemical ionization (CI)-MS m/z: 359.2442 (Calcd for $\mathrm{C}_{19} \mathrm{H}_{36} \mathrm{~N}_{2} \mathrm{O}_{2} \mathrm{Cl}$ : $\left.349.2465\left(\mathrm{M}^{+}+1\right)\right)$.

General Procedure for the Reaction of Enedione with Ph-mox-Magnesium Complex A mixture of the ligands, $\mathrm{MgI}_{2}$ and $\mathrm{I}_{2}$ in the solvent was treated under the conditions shown in Table 2. The solvent was removed and the resulting complex was dissolved in $\mathrm{CH}_{2} \mathrm{Cl}_{2}(1.0 \mathrm{ml})$ and cooled to specified reaction temperature. To this solution $8(141 \mathrm{mg}, 1 \mathrm{mmol})$ in $\mathrm{CH}_{2} \mathrm{Cl}_{2}$ $(1.5 \mathrm{ml})$ was added and stirred for $30 \mathrm{~min}$, then cyclopentadiene $(1.5 \mathrm{mmol})$ in $\mathrm{CH}_{2} \mathrm{Cl}_{2}(2.5 \mathrm{ml})$ was added slowly. After the reaction was completed the reaction mixture was quenched with water $(10 \mathrm{ml})$ and washed with $5 \%$ aqueous $\mathrm{Na}_{2} \mathrm{~S}_{2} \mathrm{O}_{3}(10 \mathrm{ml})$. The organic layer was dried and evaporated. The resulting residue was subjected to column chromatography to yield the adducts.

General Procedure for the Reaction of Enedione with Ph-mox-Copper Complex A mixture of $\mathrm{Cu}(\mathrm{OTf})_{2}(36 \mathrm{mg}, 0.1 \mathrm{mmol})$ and mox $(0.1 \mathrm{mmol})$ in $\mathrm{CH}_{2} \mathrm{Cl}_{2}(1.5 \mathrm{ml})$ was stirred for $1 \mathrm{~h}$. To this solution $8(141 \mathrm{mg}$, $1 \mathrm{mmol})$ in $\mathrm{CH}_{2} \mathrm{Cl}_{2}(1.5 \mathrm{ml})$ was added and stirred for $30 \mathrm{~min}$, then cyclopentadiene $(1.5 \mathrm{mmol})$ in $\mathrm{CH}_{2} \mathrm{Cl}_{2}(2.5 \mathrm{ml})$ was added slowly. After the reaction was completed the reaction mixture was quenched with water $(10 \mathrm{ml})$ and washed with $5 \%$ aqueous $\mathrm{Na}_{2} \mathrm{~S}_{2} \mathrm{O}_{3}(10 \mathrm{ml})$. The organic layer was dried and evaporated. The resulting residue was subjected to column chromatography to yield the adducts.

\section{References}

1) Regan A. C., J. Chem. Soc., Perkin Trans. 1, 1998, 1152-1166 (1998).

2) Juaristi E., Escalante J., Leon-Romo J. L., Reyes A., Tetrahedron; Asymmetry, 9, 715-740 (1998).

3) Ghosh A. K., Mathivanan P., Cappiello J., Tetrahedron; Asymmetry, 9, $1-45$ (1998).

4) Corey E. J., Imai N., Zhang H.-Y., J. Am. Chem. Soc., 113, 728-729 (1991).

5) Corey E. J., Ishihara K., Tetrahedron Lett., 33, 6807-6810 (1992).

6) Evans D. A., Miller S. J., Lectka T., J. Am. Chem. Soc., 115, 64606461 (1993).

7) Evans D. A., Barnes D. M., Tetrahedron Lett., 38, 57-58 (1997).

8) Davies I. W., Gerena L., Castonguay L., Senanayake C. H., Larsen R. D., Verhoeven T. R., Reider P. J., Chem. Commun., 1996, 1753-1754 (1996).

9) Brimble M. A., McEwan J. F., Tetrahedron; Asymmetry, 8, 40694078 (1997).

10) Takacs J. M., Quincy D. A., Shay W., Jones B. E., Ross II C. R., Tetrahedron; Asymmetry, 8, 3079-3087 (1997).

11) Davies D. L., Fawcett J., Garratt S. A., Russell R., Chem. Commun., 1997, 1357-1358 (1997)

12) Matt P., Pfaltz A., Angew. Chem. Int. Ed. Engl., 32, 566-568 (1993).

13) Matt P., Loiseleur O., Koch G., Pfaltz A., Tetrahedron; Asymmetry, 5, 573-584 (1994).

14) Koch G., Pfaltz A., Tetrahedron; Asymmetry, 7, 2213-2216 (1996).

15) Rieck H., Helmchen G., Angew. Chem. Int. Ed. Engl., 34, 2687-2689 (1995).

16) Honda Y., Date T., Hiramatsu H., Yamauchi M., Chem. Commun., 1997, 1411-1412 (1997).

17) Yamauchi M., Aoki T., Li M.-Z., Honda Y., Tetrahedron; Asymmetry, 12, 3113-3118 (2001).

18) Corey E. J., Imwinkelried R., Pikul S., Xiang Y. B., J. Am. Chem. Soc., 111, 5493-5495 (1989).

19) Narasaka K., Tanaka H., Kanai F., Bull. Chem. Soc. Jpn., 64, 387391 (1991).

20) Corey E. J., Sarshar S., J. Am. Chem. Soc., 114, 7938-7939 (1992)

21) Yamamoto I., Narasaka K., Chem. Lett., 1995, 1129-1130 (1995).

22) Takacs J. M., Lawson E. C., Reno M. J., Youngman M. A., Quincy D. A., Tetrahedron; Asymmetry, 8, 3073-3078 (1997).

23) Corey E. J., Matsumura Y., Tetrahedron Lett., 32, 6289-6292 (1991).

24) Evans D. A., Kozlowski M. C., Burgey C. S., MacMillan D. W. C., J. Am. Chem. Soc., 119, $7893-7894$ (1997). 